\title{
Síndrome de Burnout en estudiantes del Posgrado de Pediatría.
}

\section{Burnout Syndrome in Pediatric Posgraduate students.}

\author{
Paola Sophia Bonilla Medina*, Luis Enrique Jovel López**
}

\section{RESUMEN}

Antecedentes: El síndrome de Burnout (SB) o síndrome de desgaste profesional, descrito por primera vez en 1974 por el psiquiatra americano Herbert Freudenberger, como "estado de fatiga o frustración que se produce por la dedicación a una causa, forma de vida o relación que no produce el esperado refuerzo". El objetivo del estudio fue identificar el síndrome de Burnout en estudiantes del posgrado de pediatría de la Universidad Nacional Autónoma de Honduras en el Valle de Sula, que rotaban por el Hospital Nacional Mario Catarino Rivas durante el período de junio 2014 hasta agosto 2016. Pacientes y Métodos: Se realizó un estudio cuantitativo, descriptivo, no experimental con los médicos residentes ya descritos. Los datos se obtuvieron de un cuestionario en el cual se evaluaron características sociodemográficas, laborales y propias del síndrome de Burnout además se implementó el instrumento Maslach Burnout Inventory (MBI) adaptado al español el cual determina; cansancio emocional (CE), despersonalización (DP) y realización personal (RP), que se han clasificado en tres niveles: bajo, medio y alto Resultados: Se encuestaron 43 residentes. El 70\% (30) presentó cansancio emocional alto, 60\% (26) tenía niveles altos de despersonalización y $37 \%$ (16) presentó niveles bajos de realización personal. Se encontró que el 58\% (25) tenían síndrome de Burnout Incompleto, 7\% (3) presento Burnout completo y $35 \%$ (15) no presento el

\footnotetext{
* Residente de tercer año de Pediatría. Escuela Universitaria de Ciencias de la Salud, Universidad Nacional Autónoma de Honduras en el Valle de Sula EUCS/UNAH-VS.

**Médico Pediatra Hospital Mario Catarino Rivas.

Dirigir correspondencia a: paolasophia@gmail.com, jovel24@yahoo.es Recibido: 25 de octubre 2016 Aprobado: 04 de agosto 2017
}

síndrome. Conclusiones: Se determinó que existe el Síndrome de Burnout en los residentes de pediatría. El síndrome de Burnout incompleto fue la presentación más frecuente en más de la mitad de los estudiantes.

\section{PALABRAS CLAVE}

Agotamiento Profesional, Médicos, Asistentes de Pediatría.

\section{ABSTRACT}

Background: The clinical syndrome of Burnout (SB) or burnout syndrome was first described in 1974 by the American psychiatrist Herbert Freudenberger as "a state of fatigue or frustration that results from dedication to a cause, lifestyle or Relationship that does not produce the expected reinforcement". The objective of the study was to identify Burnout syndrome in postgraduate students of pediatrics at the National Autonomous University of Honduras in the Sula Valley, which rotated through the Hospital National Mario Catarino Rivas during the period from June 2014 to august 2016. Patients and methods: A quantitative, descriptive, non-experimental study was performed with the resident physicians already described. The data were obtained from a questionnaire in which the sociodemographic, occupational and own characteristics of the Burnout syndrome were evaluated. In addition, the Maslach Burnout Inventory (MBI) adapted to spanish was implemented, which determines; emotional exhaustion (EC), depersonalization (DP) and personal fulfillment (PR), which have been classified into three levels: low, medium and high. Results: We surveyed 43 residents. $70 \%$ (30) had high emotional fatigue, $60 \%$ (26) had high levels of depersona- 
lization and $37 \%$ (16) had low levels of personal fulfillment. It was found that $58 \%$ (25) had Incomplete Burnout Syndrome, 7\% (3) presented complete Burnout and 35\% (15) did not present the syndrome. Conclusions: It was determined that burnout syndrome exists in pediatric residents. Incomplete Burnout syndrome was the most frequent presentation in more than half of the students.

\section{KEYWORDS}

Burnout Professional, Doctors, Pediatric Assistants.

\section{INTRODUCCIÓN}

El síndrome clínico de Burnout o síndrome de desgaste profesional fue descrito por primera vez en 1974 por el psiquiatra americano Herbert Freudenberger, lo definió como «un estado de fatiga o frustración que se produce por la dedicación a una causa, forma de vida o relación que no produce el esperado refuerzo».(1-3) Para describir este patrón conductual homogéneo, Freudenberger eligió la misma palabra Burnout ("estar quemado", "consumido", "apagado") que se utilizaba también para referirse a los efectos del consumo crónico de las sustancias tóxicas de abuso. ${ }^{(4,5)}$

Es notoria la variedad de conceptos, de autores y de estudios que sobre el síndrome de Burnout se han realizado. La definición de la psicóloga Christine Maslach a partir de 1976 ha sido la más aceptada considerando que el síndrome de desgaste profesional se configura como un síndrome tridimensional. ${ }^{(2,6-8)}$ Entenderemos dicho síndrome como una patología y derivada del estrés laboral, ya que reúne los requisitos para ser llamado "síndrome", es decir, es un conjunto de signos y síntomas característicos de una enfermedad; todos ellos agrupados en tres dimensiones: agotamiento emocional (cansancio físico y mental), despersonalización (sentimientos de impotencia y desesperanza) y falta de realización personal en el trabajo (evitación, desmotivación, baja autoestima, etc.); debida a una exposición gradual y continuada de por lo menos 6 meses, a los estresores que el trabajador percibe como negativos para su salud, existentes en el medio que rodea al trabajador. ${ }^{(6,9,10)}$

El objetivo principal de este estudio fue identificar el síndrome de desgaste profesional o Burnout en estudiantes del posgrado de pediatría de la Universidad Nacional Autónoma de Honduras en el Valle de Sula (UNAH-VS), que rotaban por el Hospital Nacional Mario Catarino Rivas durante el período de junio 2014 hasta agosto 2016.

\section{PACIENTES Y MÉTODOS}

Estudio cuantitativo, descriptivo, no experimental, realizado en estudiantes del posgrado de pediatría de la Universidad Nacional Autónoma de Honduras en el Valle de Sula, que rotaban por el Hospital Nacional Mario Catarino Rivas (HNMCR), durante el período de junio 2014 a agosto del 2016. Se incluyeron un total de 43 médicos residentes de pediatría. Los datos fueron obtenidos mediante un cuestionario en el cual se interrogó las características sociodemográficas de la población y características laborales, además se aplicó el cuestionario Maslach de 1986 que es el instrumento más utilizado en todo el mundo para evaluar el desgaste profesional, esta escala tiene una alta consistencia interna y confiabilidad cercana al $90 \%$, está constituido por 22 ítems que se presentan a consideración del interrogado en una escala de Likert, en forma de afirmaciones, con 7 categorías (van desde "nunca" que tiene valor de cero hasta "todos los días" con valor de 6), sobre los sentimientos y actitudes del profesional en su trabajo y hacia los pacientes. Se establecieron las tres dimensiones del Burnout: cansancio emocional (CE), despersonalización (DP) y realización personal (RP), que se han clasificado en tres niveles: bajo, medio y alto, de acuerdo con los puntos RP $>=40$ 34-39 
$<=33$. En las tres escalas la puntuación mínima alcanzable es 0 , mientras que la máxima es 54 para $C E, 30$ para DP y 48 para RP.

El Síndrome de Burnout completo: se presenta en aquellos individuos con niveles altos de cansancio emocional y despersonalización y niveles bajos de realización personal.

El Síndrome de Burnout incompleto: incluye los individuos que presentan niveles altos de cansancio emocional y despersonalización con niveles medios de realización personal. ${ }^{(4)}$

El análisis estadístico se realizó en una base de datos electrónica creada en el programa informático SPSS Versión 18; se determinaron medidas de tendencia central, tablas de frecuencias, porcentajes y tablas de contingencia; para generar su análisis. Los gráficos fueron realizados en Excel Versión 2016.

\section{RESULTADOS}

\section{Características socio-demográficas de la población}

De los 43 residentes encuestados, la media, moda y mediana de la edad fue de 29 años deviación estándar \pm 2.4 con edad mínima de 26 y máxima de 44 años. Se encontró que $58 \%$ (25) eran masculinos y $42 \%$ (18) femeninos. En relación al estado civil; 55\% (24) eran solteros, $33 \%$ (14) estaban casados(as) y $12 \%$ (5) en unión libre. Del total de la población encuestada: $26 \%$ (11) tenían hijos y $74 \%$ (32) no tenían.

\section{Características profesionales de la pobla- ción}

En la población encuestada el horario de trabajo se mantuvo en el rango de 6:30 am hasta las 17:00 horas, del total encuestados ninguno consumía psicofármacos; se interrogó acerca de enfermedades crónicas de los cuales el 7\% (3) presentaron enfermedades crónicas y el 93\% (40) no lo presentaron. En la muestra se- leccionada se realizó la distribución en porcentajes de la interacción durante la jornada diaria, el médico interaccionaba con pacientes y familiares; esto se puede ver reflejado en la tabla No. 1.

Tabla No. 1: Porcentaje de interacción de los residentes con pacientes y sus familiares.

\begin{tabular}{|c|c|c|c|c|}
\hline $\begin{array}{c}\text { \% que pasa } \\
\text { con } \\
\text { pacientes } \\
\text { y sus } \\
\text { familiares }\end{array}$ & $\begin{array}{l}25 \%- \\
50 \%\end{array}$ & $\begin{array}{l}50 \%- \\
75 \%\end{array}$ & $\begin{array}{c}\text { Más de } \\
75 \%\end{array}$ & $\begin{array}{c}\text { Total } \\
\text { general }\end{array}$ \\
\hline Femenino & 3 & 4 & 11 & 18 \\
\hline Masculino & 3 & 11 & 11 & 25 \\
\hline Total & 6 & 15 & 22 & 43 \\
\hline Porcentaje & $14 \%$ & $35 \%$ & $51 \%$ & $100 \%$ \\
\hline
\end{tabular}

Fuente: Instrumento Maslach Burnout Inventory (MBI) adaptado al español en Hospital Nacional Dr. Mario Catarino Rivas San Pedro Sula. UNAH. 2014- 2016.

Se determinó la percepción de pacientes atendidos por los residentes el cual puede observarse en la tabla No. 2.

Tabla No. 2: Percepción del número de pacientes atendidos.

\begin{tabular}{cccc}
\hline \hline $\begin{array}{c}\text { Pacientes } \\
\text { que atiende }\end{array}$ & Adecuado & Excesivo & Total \\
\hline Femenino & 2 & 16 & 18 \\
Masculino & 2 & 23 & 25 \\
Total & 4 & 39 & 43 \\
Porcentaje & $9 \%$ & $91 \%$ & $100 \%$ \\
\hline \hline
\end{tabular}

Fuente: Instrumento Maslach Burnout Inventory (MBI) adaptado al español en Hospital Nacional Dr. Mario Catarino Rivas San Pedro Sula. UNAH. 2014- 2016. 
En la población estudiada se encuestó acerca de la valoración que estos sentían en su vida laboral donde podemos observar que la valoración de estos por sus pacientes: el 72\% (31) se sentía valorado y el $28 \%$ (12) no se sentía valorado por sus pacientes, se determinó la valoración por los familiares de los pacientes de los cuales el $58 \%$ (25) se sentía valorados por estos y el $42 \%$ (18) no se sentía valorado. Además, con respecto a la valoración por sus compañeros el 91\% (39) se sentía valorado por sus compañeros y el 9\% (4) no se sentía valorado por estos. Posteriormente se determinó la valoración por los superiores de los cuales el 70\% (30) se sentía valorado por sus superiores y el $30 \%$ (13) no.

Se encontró que 75\% tenían buena situación económica. (Ver tabla No. 3).

Tabla No. 3: Valoración de su situación económica.

\begin{tabular}{lcc}
\hline \hline $\begin{array}{c}\text { Situación } \\
\text { económica }\end{array}$ & Frecuencia & Porcentaje \\
\hline Mala 1-3 & 1 & $2 \%$ \\
Regular 4-6 & 10 & $23 \%$ \\
Buena 7-10 & 32 & $75 \%$ \\
Total & 43 & $100 \%$ \\
\hline \hline
\end{tabular}

Fuente: Instrumento Maslach Burnout Inventory (MBI) adaptado al español en Hospital Nacional Dr. Mario Catarino Rivas San Pedro Sula. UNAH. 2014- 2016.
Se determinó a partir de una escala de satisfacción según la experiencia profesional en la residencia, que $96 \%$ tienen experiencia profesional muy satisfactoria. (Ver tabla No. 4).

Tabla No. 4: Valoración global de su experiencia profesional.

\begin{tabular}{lcc}
\hline \hline $\begin{array}{l}\text { Experiencia } \\
\text { Profesional }\end{array}$ & Frecuencia & Porcentaje \\
\hline $\begin{array}{l}\text { Nada } \\
\text { Satisfactorio } 1-3\end{array}$ & 1 & $2 \%$ \\
Satisfactorio $4-6$ & 1 & $2 \%$ \\
$\begin{array}{l}\text { Muy } \\
\text { Satisfactorio 7-10 }\end{array}$ & 41 & $96 \%$ \\
Total & 43 & $100 \%$ \\
\hline \hline
\end{tabular}

Fuente: Instrumento Maslach Burnout Inventory (MBI) adaptado al español en Hospital Nacional Dr. Mario Catarino Rivas San Pedro Sula. UNAH. 2014- 2016.

\section{Factores relacionados con el Síndrome de} Burnout.

En relación al cansancio emocional, se encontró que era alto en 30 (70\%) de los Médicos Residentes (14 del sexo femenino y 16 masculinos). (Ver gráfica No. 1) 
Gráfica No. 1: Grado de cansancio emocional según sexo.

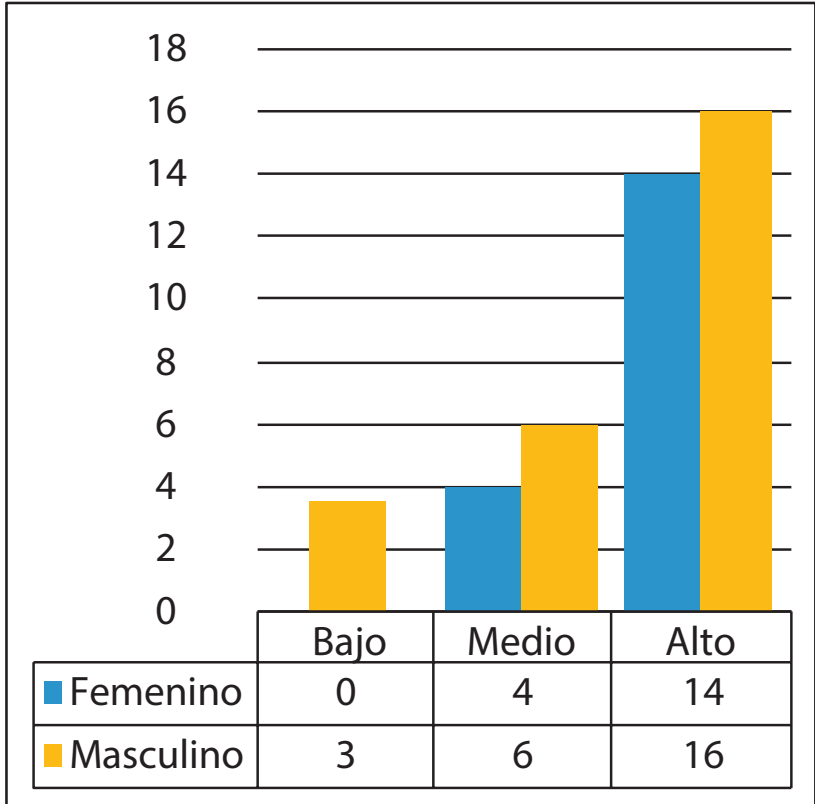

Fuente: Instrumento Maslach Burnout Inventory (MBI) adaptado al español en Hospital Nacional Dr. Mario Catarino Rivas San Pedro Sula. UNAH. 2014- 2016.

En relación a la despersonalización se determinó que el 60\% (26) tenía niveles altos de despersonalización ( 10 del sexo femenino y 16 masculinos) esto se aprecia en la gráfica No. 2.

\section{Gráfica 2: Grado de despersonalización.}

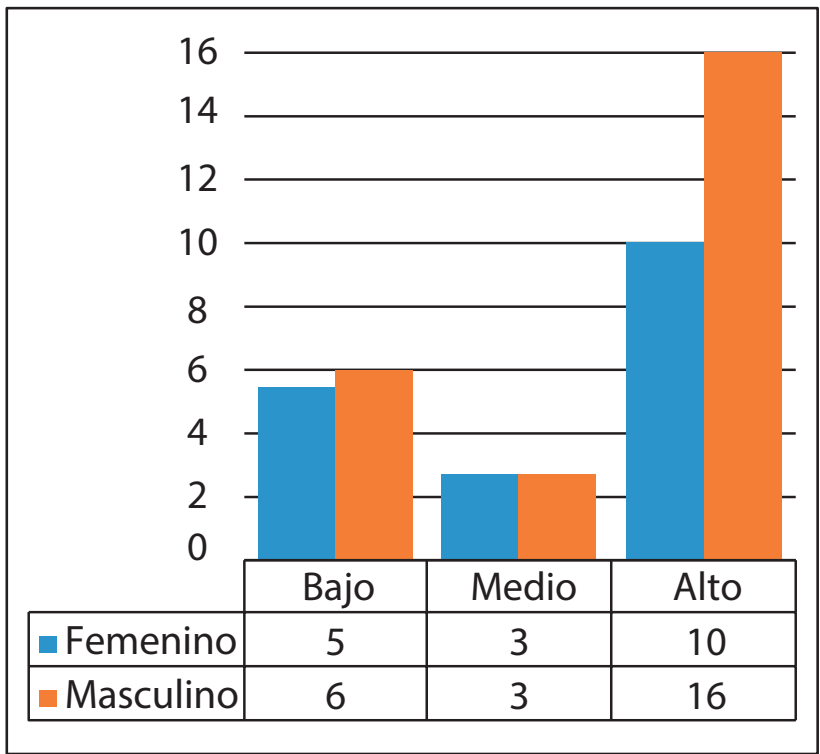

Fuente: Instrumento Maslach Burnout Inventory (MBI) adaptado al español en Hospital Nacional Dr. Mario Catarino Rivas San Pedro Sula. UNAH. 2014- 2016.
En la realización personal el 37\% (16) presento niveles bajos de realización personal (8 del sexo femenino y 5 masculinos) esto se ve reflejado en la gráfica No. 3.

Gráfica No. 3: Grado de realización personal.

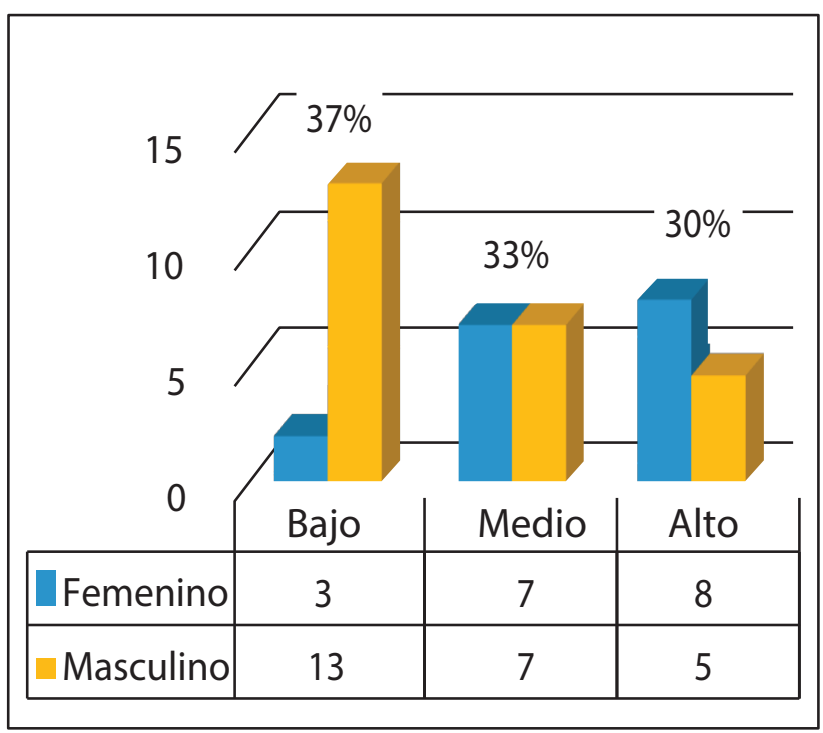

Fuente: Instrumento Maslach Burnout Inventory (MBI) adaptado al español en Hospital Nacional Dr. Mario Catarino Rivas San Pedro Sula. UNAH. 2014- 2016.

Además, el $65 \%$ de los encuestados presentó algún grado de síndrome de Burnout, esto se refleja en la gráfica No. 4.

\section{Gráfica No. 4: Grado de Burnout en residen- tes del posgrado de pediatría.}

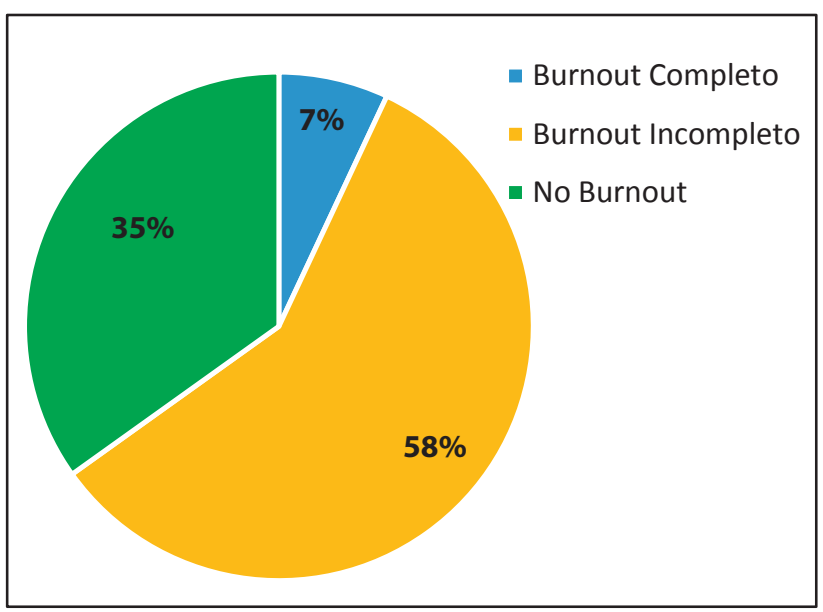

Fuente: Instrumento Maslach Burnout Inventory (MBI) adaptado al español en Hospital Nacional Dr. Mario Catarino Rivas San Pedro Sula. UNAH. 2014- 2016. 
Se encontró que 43\% (12) de los residentes, con síndrome de Burnout tenían 29 años. Se identificó que de los residentes con síndrome de Burnout, $71 \%$ (20) eran del sexo masculino.

(Ver tabla No. 5).

Tabla No. 5: Características de los Médicos Residentes, con Síndrome de Burnout.

\begin{tabular}{|c|c|c|c|}
\hline $\begin{array}{l}\text { Carac- } \\
\text { terística }\end{array}$ & Categoría & $\begin{array}{l}\text { Frecu- } \\
\text { encia }\end{array}$ & $\begin{array}{c}\text { Porcen- } \\
\text { taje }\end{array}$ \\
\hline \multirow[b]{5}{*}{ Edad } & 26 años & 3 & $10.5 \%$ \\
\hline & 28 años & 3 & $10.5 \%$ \\
\hline & 29 años & 12 & $43 \%$ \\
\hline & 30 años & 8 & $29 \%$ \\
\hline & 31 años & 2 & $7 \%$ \\
\hline \multirow[b]{3}{*}{ Sexo } & TOTAL & 28 & $100 \%$ \\
\hline & Femenino & 8 & $29 \%$ \\
\hline & Masculino & 20 & $71 \%$ \\
\hline \multirow[b]{4}{*}{ Estado Civil } & TOTAL & 28 & $100 \%$ \\
\hline & Solteros & 16 & $57 \%$ \\
\hline & Casados & 8 & $29 \%$ \\
\hline & Unión libre & 4 & $14 \%$ \\
\hline \multirow[b]{3}{*}{ Tienen hijos } & TOTAL & 28 & $100 \%$ \\
\hline & Tiene hijos & 8 & $29 \%$ \\
\hline & No tiene hijos & 20 & $71 \%$ \\
\hline \multirow{5}{*}{$\begin{array}{c}\text { Año de } \\
\text { residencia }\end{array}$} & TOTAL & 28 & $100 \%$ \\
\hline & Primer año & 8 & $29 \%$ \\
\hline & Segundo año & 14 & $50 \%$ \\
\hline & Tercer año & 6 & $21 \%$ \\
\hline & TOTAL & 28 & $100 \%$ \\
\hline
\end{tabular}

Fuente: Instrumento Maslach Burnout Inventory (MBI) adaptado al español en Hospital Nacional Dr. Mario Catarino Rivas San Pedro Sula. UNAH. 2014- 2016.

\section{DISCUSIÓN}

En cada etapa de la carrera profesional, el médico está sometido a tensiones y pruebas que supera con mayor o menor dificultad psicológica. Partiendo de lo anterior, podemos considerar que los años de la residencia médica son especialmente vulnerables dado que es el período en el que se produce la transición de las expectativas idealistas a la práctica cotidiana y se aprende que las recompensas personales, económicas y profesionales, no son ni las prometidas ni las esperadas. ${ }^{(11)}$
En este estudio, se encontró que el $74 \%$ de los residentes no tiene hijos y el $26 \%$ tienen hijos, dichos datos son similares con un estudio realizado en el Hospital General Regional el cual representa el $22.2 \%$ de los residentes no tenían hijos; ${ }^{(12)}$ pero difieren con otro estudio realizado en residentes de neurología en México donde el 9\% tenía hijos y el $91 \%$ aún no. ${ }^{(13)}$ En la muestra seleccionada se realizó la distribución en porcentajes de la interacción durante la jornada diaria el médico interaccionaba con pacientes y familiares de estos donde el $51 \%$ interactuaba más del 75\% del día, dichos resultados son diferentes a otros estudios donde el 78,6\% laboraba más de 18 horas al día este estudio fue realizado en una muestra de 138 residentes de especialidades médico quirúrgicas de la Universidad Militar Nueva Granada, Bogotá, Colombia. ${ }^{(14)}$

Se realizó una escala de satisfacción en el cual la percepción de la valoración por sus pacientes $72 \%$ se siente valorado y solo $28 \%$ no se sentía valorado por sus pacientes estos datos son similares a un estudio España ${ }^{(15)}$ donde se entrevistó a 132 residentes y el $18.1 \%$ no se sentía valorado por sus pacientes. En este estudio el $58 \%$ se siente valorado por familiares de sus pacientes y el $42 \%$ no lo percibe así estos datos son diferentes al estudio anteriormente mencionado ya que el $28.2 \%$ no se sentía valorado por familiares de pacientes es decir que es más alta la percepción de no valoración en este estudio.

Se determinó la valoración que percibían los residentes por sus compañeros donde el $9 \%$ de los residentes no se sentía valorado por sus compañeros, estos datos son positivos ya que en el estudio realizado en España el 27.6\% de los residentes no se sentía valorado por sus compañeros. Además, se realizó la percepción de valoración de los residentes por sus superiores donde el 30\% no lo percibía así, estos resultados son casi similares al estudio antes men- 
cionado donde $47.2 \%$ no se sentía valorado por sus superiores. ${ }^{(15)}$

Se realizó una escala de satisfacción de la experiencia profesional de los residentes en la cual el $96 \%$ lo determinó como muy satisfactorio, estos resultados son superiores a los encontrados en el estudio en residentes adscritos en diferentes hospitales de México en el que el $57 \%$ refirió la experiencia laboral como buena. ${ }^{(16)}$

Para la valoración de su situación económica el $75 \%$ estableció como buena su situación económica, estos resultados son diferentes al estudio realizado en residentes de España donde catalogaron su situación económica como regular. ${ }^{(15)}$

Se determinó el grado de cansancio emocional el cual estaba en niveles altos $70 \%$, para el grado de despersonalización el nivel alto fue $60 \%$ dichos resultados concuerdan con estudios realizados en Hospital Privado de Córdoba, Argentina donde el nivel fue $75.5 \%$ y para el grado de despersonalización fue $79.9 \%$ respectivamente. Además, se estableció el grado de realización personal donde la baja realización personal fue del $37 \%$ estos resultados concuerdan con el estudio realizado en el Hospital de Córdoba donde la baja realización personal correspondió al 33.6\%. ${ }^{(17)}$

En el presente estudio por medio del instrumento Maslach Burnout Inventory se determinó que el $65 \%$ de los residentes presentaban algún grado del síndrome de Burnout, de los cuales solo el 7\% presento un síndrome de Burnout completo dichos resultados concuerdan con un estudio realizado en el Hospital Privado de Córdoba, Argentina, donde el 5\% presento síndrome de Burnout completo, en este último estudio no se determinó el síndrome de Burnout incompleto. ${ }^{(18)}$ Existe otros lugares donde la muestra fue de 138 resi- dentes de las diferentes especialidades médico quirúrgicas de la Universidad Militar Nueva Granada, Colombia el $12,6 \%$ de los residentes presentó un alto nivel de Burnout. $^{(14)}$

La edad más frecuente para el síndrome de Burnout fue a los 29 años estos datos concuerdan con los obtenidos en el Hospital Nacional Dos de Mayo, donde se realizó un estudio con 96 residentes encontrando un mayor porcentaje de síndrome de Burnout $(56,1 \%)$ en los residentes de 31 años. Así mismo se encontró que todos los residentes que presentaban 41 años o más no lo presentaban. En este estudio se determinó la presencia del síndrome de Burnout según sexo donde el $29 \%$ correspondió al sexo femenino y el $71 \%$ al sexo masculino, estos datos son similares a estudios realizados en Perú en el cual la distribución del síndrome de Burnout es del $29 \%$ para el sexo femenino y del $70.7 \%$ para el sexo masculino. ${ }^{(18)}$

En los encuestados se determinó la presencia del síndrome de Burnout según el estado civil donde el $57 \%$ de los solteros presentó dicho síndrome, el $29 \%$ de los casados y solo el $14 \%$ en unión libre presentó síndrome de Burnout, estos datos son comparables con el estudio realizado a 143 médicos residentes de México en el cual el síndrome de Burnout respecto al estado civil hubo una diferencia porcentual mínima entre solteros y casados, con una frecuencia de 47.9 y $52.9 \%$ respectivamente. ${ }^{(19)}$

Se estableció el síndrome de Burnout y la presencia o no de hijos y el 73\% de los residentes que tienen hijos presentan algún grado de síndrome de Burnout, estos resultados son preocupantes debido a que en otros estudios solo un $22,2 \%$ de los que presentaban síndrome de Burnout tenía hijos. ${ }^{(18)}$

En la muestra encuestada se estratificó la presencia del síndrome de Burnout según el 
año de residencia en donde los residentes de segundo año presentaron el mayor porcentaje del síndrome de Burnout 50\%, esto puede explicar que el primer año es un proceso adaptación, segundo año se espera del residente más conocimiento y más capacidad en la resolución de problemas y esto desde el inicio del segundo año, tercer año ya conoce sus capacidades y limitaciones siente predilección por determinada área de la especialidad y puede delegar responsabilidades. Estos datos difieren a estudios realizados en Paraguay donde los residentes de primer y tercer año resultaron ser los más afectados, seguido de los del segundo año de residencia; siendo Pediatría, Gineco- Obstetricia y Cirugía General los afectados con residentes con síndrome de Burnout. ${ }^{(20)}$ El síndrome de Burnout puede ser bien manejado por todos aquellos que trabajan en el ámbito de la salud si fuesen informados y prevenidos sobre el mismo, también enseñándoles como tolerar o deshacerse de todas aquellas situaciones que generasen emociones encontradas con uno mismo o con las diversas personas (directivos, colegas, personal de enfermería, familiares, amigos, etc.) que tratan diariamente. Teniendo en cuenta que el sistema de residencias médicas para las diferentes especialidades es la mejor manera de adquirir conocimientos y práctica, hemos analizado que la mejor propuesta seria enfatizar en la ayuda al joven profesional y futuro especialista con recursos como talleres de autoayuda o la implementación de servicios psicopedagógicos exclusivos con visitas frecuentes para monitorear las diferentes situaciones.

En conclusión, estos resultados podrían explicar que las condiciones laborales para los profesionales en formación en las diferentes especialidades médicas muchas veces no son las adecuadas; no respetan los ritmos circadianos biológicos, lo cual aumenta la vulnerabilidad de padecer afecciones psíquicas relacionadas al estrés, como el denominado "Síndrome de Burnout", entre otros, y consecuentemente disfunciones en el plano social y familiar del médico residente; situación que repercute en forma negativa directa e indirectamente en la calidad de atención de los pacientes. 


\section{BIBLIOGRAFÍA}

1. Kaschka WP, Korczak D, Broich K. Burnout: a fashionable diagnosis. DtschArztebl Int. 2011; 108(46): 781-7.

2. Castañeda Aguilera E, García de Alba García JE. Prevalencia del síndrome de agotamiento profesional (Burnout) en odontólogos de una institución de la salud,en la ciudad de Guadalajara, México, 2012. RevFac de OdontolUniv de Antioq. 2013; 24(2): 267-288.

3. Carlin M, Garcés de los Fayos Ruiz EJ. El síndrome de burnout: evolución histórica desde el contexto laboral al ámbito deportivo. Anales de psicología; 2010; 26(1): 169-180.

4. Ramón Z, María, J. Identificación del Burnout en profesionales de la salud y factores relacionados con este fenómeno, en el Hospital Luis Mosco Zambrano de la ciudad de Piñas, Hospital Humberto Molina de la ciudad de Zaruma y Dispensario del IESS de Zaruma. Durante el año 2011-2012; 2012. [Tesis].Zaruma: La Universidad Tecnica Particular de Loja; 2012.

5. Zhang $Y$, Feng $X$. The relationship between job satisfaction, burnout, and turnover intention among Physicians from urban state-owned medical Institutions in Hubei, China: a cross-sectional Study. BMC HealthServ Res [Internet]. 2011 [Consultado el 5 de mayo 2016]. Disponible en: http://www. biomedcentral.com/1472-6963/11/235.

6. Caballero Martín MA, Bermejo Fernández F, Nieto Gómez R, y Caballero Martínez F. Prevalencia y factores asociados al burnout en un área de salud. Aten Primaria. 2001; 27(5): 313-17.

7. Meana MA. Síndrome de burnout: su preva- lencia en una población de médicos en programas intensivos de capacitación en la especialidad de Clínica Médica. [Tesis doctoral]. Argentina: Universidad Nacional de la Plata; 2009.

8. Quaas C. Diagnóstico de burnout y técnicas de afrontamiento al estrés en profesores universitarios de la quinta región de chile.Psicoperspectivas. 2006; 5(1): 65-75.

9. Blandin J, Martínez D. Estrés laboral y mecanismos de afrontamiento: su relación en la aparición del síndrome de burnout en médicos residentes del Hospital Militar Dr. Carlos Arvelo. Arch Venez Psiquiatr Neurol. 2005; 51(104): 12-15.

10. Aranda Beltrán C. Diferencias por sexo, síndrome de burnout y manifestaciones clínicas, en los médicos familiares de dos instituciones de salud, Guadalajara, México. Revcostarric salud pública. 2006; 29(15): 1-7.

11. Balcázar Rincón LE, Montejo Fraga LF, Ramírez Alcántara YL. Prevalencia del síndrome de desgaste profesional en médicos residentes de un hospital de Mérida, Yucatán, México. Aten Fam. 2015; 22(4): 111-4.

12. Camacho-Ávila A, JuárezGarcía A, Arias Galicia F. Síndrome de burnout y factores asociados en médicos estudiantes. CiencTrab [Internet]. 2010 [Consultado el 2 marzo 2016]; 35. Disponible en: http://fac torespsi cosociales.com/segundoforo/sim posio/ca macho-juarez-arias.pdf

13. Velásquez-PérezL, Colin-Piana RF, González-González M. Afrontando la residencia médica: depresión y burnout. GacMédMéx. 2013; 149; (2): 183-195. 
14. Paredes G OL, Sanabria-Ferrand PA. Prevalencia del síndrome de burnout en residentes de especialidades médico quirúrgicas, su relación con el bienestar psicológico y con variables sociodemográficas y laborales. RevFac.Méd. 2008;16(1):25-32.

15. Grau-Martín A, Suñer-Soler R. Síndrome de burnout y residentes: reflexiones sobre la magnitud del fenómeno. RevClín Esp. 2011; 211(2): 112-13.

16. Ríos Puertas AL,Athié García JM, Martínez Rosete VA. Síndrome de burnout (quemado) en médicos residentes adscritos a hospitales del Grupo Ángeles: HA Clínica Londres, HA Lomas, HA Metropolitano, HA México, HA Mocel y HA Pedregal. Acta Médica Grupo Ángeles. 2015;13(2):69-81.
17. Ponce LB, Gavotti GC, Ferreyra L, Flores M. Síndrome de Burnout en médicos residentes del Hospital Privado. Año 2013. Experiencia Médica. 2016;33(1):8-11.

18. Mendoza SaldivarMK. Prevalencia del síndrome de burnout en residentes de especialidades clínicas y quirúrgicas del Hospital Nacional dos de mayo, noviembre 2015. [Tesis]. Lima: Facultad de Medicina Humana, Universidad Ricardo Palma; 2016.

19. ArayagoR, González Á, Limongi M, Guevara $H$. Síndrome de Burnout en residentes y especialistas de anestesiología. Salus. 2016;20(1):13-21.

20. Alfonso Recalde SS, Ferrera Gaona MI, Díaz Reissner CV. Síndrome de burnout en residentes de especialidades médicas. Hospital Central Dr. Emilio Cubas of IPS. Eureka. 2015; 12 (1): 25-34. 